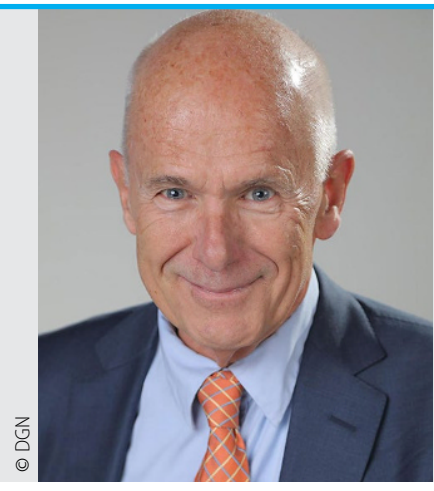

Prof. Dr. Peter Berlit

Deutsche Gesellschaft für Neurologie

Reinhardtstr. 27 C, 10117 Berlin, Deutschland

berlit@dgn.org

\title{
Facharzt-Training Neurologie
}

\section{Fallbezogenes Lernen anhand der neuen Musterweiterbildungsordnung}

Die Neurologie ist ein klinisches Fach mit einem kontinuierlichen Wissenszuwachs, großer Innovationskraft und enormer Detailfülle. Trotz der bemerkenswerten Fortschritte in der apparativen Diagnostik und individualisierten Therapie bleibt die richtige Einordnung der Patientenanamnese und des klinischen Untersuchungsbefundes jedoch die Basis des Fachs. Vom Symptom über das Syndrom ist es möglich, eine erste Eingrenzung der Lokalisation vorzunehmen und eine Arbeitsdiagnose zu erstellen. Ausgehend davon werden gezielt neurophysiologische, laborchemische und bildgebende Verfahren eingesetzt, um die Verdachtsdiagnose zu verifizieren oder zu falsifizieren. Im Alltag von Klinik und Praxis, insbesondere im Notdienst, müssen die entsprechenden Entscheidungsschritte rasch erfolgen. Und gerade dies bereitet dem Anfänger im Beruf oft Sorgen. Auch bei der enormen Arbeitsverdichtung und engen Taktung im klinischen Alltag auf der Station und in der neurologischen Praxis muss das Wissen parat stehen, um unseren Patienten gerecht werden $\mathrm{zu}$ können. Sowohl die Umfragen unter unseren jungen Neurologen als auch das von unserer Präsidentin Frau Professorin Christine Klein angestoßene ReImagine-Medicine-Projekt zeigten, dass die Aus- und v. a. die Weiterbildung eines der wichtigsten Themen für unsere Mitglieder sind.

Dem versuchen wir, mit unserer Zeitschrift DGNeurologie Rechnung zu tragen, indem wir Krankheitsbilder in Formaten wie SOP („standard operating procedure“), ZOOM und CME („continuing medical education“) darstellen und Lerninhalte darüber hinaus in verschiedenen kasuistischen Rubriken regelmäßig anbieten. Die Rückmeldungen, die wir von den Lernenden, aber auch den Lehrenden erhalten, zeigen, dass v. a. das fallbezogene Lernen besonders effektiv ist.

\section{Kompaktes Wissen für die Facharztprüfung}

Dieser Effektivität des fallbezogenen Lernens wollen wir nun zusätzlich Rechnung tragen, indem wir gezielt die Inhalte der neuen
Musterweiterbildungsordnung kasuistisch aufbereiten, um das für den Facharzt relevante Wissen benutzerfreundlich zum Lernen und Repetieren anzubieten. In den Händen halten Sie die erste Sonderausgabe Facharzt-Training Neurologie mit 32 Fallbeispielen. Geplant sind zunächst 64 Fälle zu den wichtigsten neurologischen Krankheitsbildern in 2 Sonderheften. Diese sollen thematisch das Wissen der Facharztprüfung im Wesentlichen abdecken. In Vorbereitung auf diese könnten dann noch Spezialbereiche wie die neurologische Intensivmedizin und die apparativen Methoden mögliche weitere Themenbereiche sein.

\section{Sonderhefte für alle DGN-Mitglieder}

In der Zeitschrift DGNeurologie finden sich die Inhalte illustriert mit Bildern. Alle Mitglieder der DGN (Deutsche Gesellschaft für Neurologie) erhalten im Rahmen ihrer Mitgliedschaft die gedruckten Sonderhefte der Zeitschrift DGNeurologie, FacharztTraining Neurologie, zugesandt.

Ich bedanke mich bei allen Autoren, die sich an diesem Projekt so engagiert beteiligen und aus ihrem Spezialgebiet instruktive Patientenbeispiele beitragen; und bei Frau Wasser und Frau Weber vom Springer Medizin-Verlag, die dieses Projekt so effektiv begleiten.

Unseren Leserinnen und Lesern wünsche ich eine spannende und v. a. effektive Lektüre - lassen Sie mich wissen, was Sie von diesem besonderen Angebot Ihrer DGN halten.

Mit herzlichen Grüßen

Ihr Peter Berlit

Interessenkonflikt. P. Berlit gibt an, dass kein Interessenkonflikt besteht. 